\title{
VI. Ueber die Einwirkung des Jodoforms auf das Wachsthum und die Virulenz der Milz- brandbacillen.
}

\section{Von Dr. Emil Senger.}

\author{
(Schluss aus No. 33.)
}

Prüfung der Virulenz der Bacillen.

Entsprechend den Versuchen im Reagenzglase hinsichtlich des 'Wachsthums und der Fortpflanzungsfähigkeit wurde auch die Virulenz der Bacillen, welche mit Jodoform in Berührung gebracht waren, geprüft. Diese Versuche wurden zu gleicher Zeit angestellt; nur der Uebersichtlichkeit wegen ist das Resultat in Bezug auf Wachsthum etc. aus verschiedenen Versuchen oben zusammengestellt.

Für alle weiteren Experimente erwähne ich hier Folgendes. Unter Impfung bei Mäusen ist immer die subcutane Application nach Anlegung einer Hauttasche am Schwanzende gemeint. Zunächst wurde die Reinheit einer Cultur constatirt, dann die Hauttasche angelegt und mittelst einer dicken Platin-Oese Anthrax und Jodoform zusammen in dieselbe gebracht. Es liess sich nie vermeiden, dass Jodoform mitgenommen wurde, weil man in der Gelatine die Anthraxcultur nicht erkennen konnte. Der Rest der Impfmasse an der Nadel wurde auf ein Deckgläschen verrieben, und nach der Impfung Bacillen nachgewiesen, wo nicht, wurde der Versuch wiederholt. Es ist also mit absoluter Sicherheit Anthrax in die Tasche gekommen, allerdings immer auch eine Spur von Jodoform. Mitunter wurde auch die verflïssigte Gelatine mit dem Jodoform geschüttelt, so dass eine gelbe Emulsion entstand und hiervon wurde geimpft.

Die nachfolgende Tabelle giebt das Resultat der Impfungen. Unter Jodoform-Anthrax-Gelatine verstehe ich eine Mischung von ca. $1^{1 / 2} \mathrm{~g}$ Jodoform mit einer ursprünglich fast ganz verflüssigten Anthrax-Gelatinecultur; unter Sublimat-Anthrax-Gelatine verstehe ich die Hinzufügung von 1 g Sublimat $(1: 2000)$ zu einer ebensolchen Anthraxcultur. Der Ausdruck 1, 2, 3 Tage alte Cultur heisst, dass die Impfung $1,2,3$ Tage nach der angestellten Mischung vorgenommen ist. Vor jeder Mischung wurde jede Cultur auf ihre Virulenz an Mäusen geprüft. Die Mäuse starben innerhalb 18-24 Stunden an Milzbrand. Obgleich das Wachsthumsresultat schon vorher mitgetheilt ist, füge ich es dennoch der Uebersichtlichkeit wegen bei und bemerke nochmals, dass von dem Originalgläschen zu gleicher Zeit die Impfung einer Maus und (zur Wachsthumsprüfung) die Impfung dreier frischen Gelatinegläschen vorgenommen wurde.

\section{Tabelle.}

Jodoform-Anthrax-Gelatine. Viruleuprïfung Wachsthumsanf Mäuse. prü fung immer
auf 3 Gläschen.

Sublimat-Anthrax-Gelatine. (Contr.) anf Manse.

li.

1) 1 Tag alte Cul-
tur wird geimpft.

Versuchsreihe (8 Experimente).

Tod nach $3 \mathrm{~T}$ agen. \begin{tabular}{c|c} 
Impfung in 3 & $1 \mathrm{Tag}$ alte Cultur \\
Gläschen steril; & wird geimpft, Maus
\end{tabular} trotz zunehmen- hleibt ges ulud.

der Verflüssigrung im Originalgläschen.

Il. Versuchsreihe (8 Experimente).

2) 2 Tage alte Cul- steril; 1 Glas 2 Tage alte Cultur

tur wird geimpft. durch Coccen geimptt. Maus hleibt

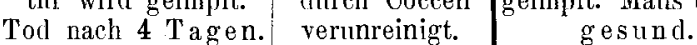

$\left.3^{3}\right) 1$ Tag alte CulIII. Versuchsreihe (8 Experimente). tur wirl geimpft. Im

Schwanzblut der lo-

benden Maus am

4. Tage viele $\mathrm{Ba}$ -

cillen. Abends am

4. Tage todt an Milz-

brand. Tod nach

4 Tagen.

V. Versuchsreihe (s Experimente)

$\left.4^{3}\right) 2$ T'age alte Abimpfung steril IV. Maus gesund

Cnl ltur geimpft. Tod an 3 Gläschen geblieben.

an Milzhrand nach 1 Monat beo-

$1 \frac{1}{2}$ Tag.

bachtet.

V. Versuchsreihe (8 Experimente).

$\left.5^{3}\right) 4$ Tage alte wie vorher.

Wachsthum immer auf 3 Gläschen.

Impfung steril bei fortschreitender Verflüssigutu im Originalglas.

steril.

steril.

steril.

do. 
VI. Versuchsreihe (8 Experimente).

\begin{tabular}{|c|c|}
\hline $\begin{array}{c}6^{2} \text { ) } 4 \text { Tage alte } \\
\text { Cultur geimpft. Ge- } \\
\text { schüttelt und Emul- } \\
\text { sion hergestellt. } 2 \\
\text { Tage lang sehr krank, } \\
\text { kleine Augen, strup- } \\
\text { pige Haare keine } \\
\text { Fresslust, darauf er- } \\
\text { holt sie sich und } \\
\text { bleibt, 16 Tage } \\
\text { beobachtet, gesund. }\end{array}$ & steril. \\
\hline
\end{tabular}

VII. Versuchsreihe (8 Experimente).

7) 5 Tage alte

Cultur geimpft.

1 Jag darauf krank,

an 4. und 10. Tage

keine Bacillen im

Schwanzblut zu con-

statiren, sie bleibt

gesund.

83) 7 Tage alto

Cultur geimpft.

(15 Tage beobachtet)

bleibt gesund.

\section{$5 \mathrm{~T}$ ag e alte Cultur ge- Veruureinigt impft, bleibt g esund. 2 Gläschell. Voll weiteren Sub- limat-Impfungen wird Abstand ge- nommell.}

VIII. Versuchsreihe.

$$
\mid
$$

IX. Versuchsreihe.

7 Tage alte Cultur wird geimpft, sehr reichlich. Nach $1 \frac{1}{2} \mathrm{Tagen}$ Tod unter den Erscheinungen des Wahnsinns. Die Maus schiesst Kobold, springt wild umher, gräht sich dann wieder in die Sägespäne etc. Im Blut des Herzens, der Jeber, Milz etc. keine Bacillen zu finden. Weisse Blutkörperchen scheineu vermehrt. Der Tod ist also nicht als Milzbrand-, sondern als Jodoformtod anzusehen. Da die Wahnsinnserscheinungen mir bei der Maus neu waren, so wurde

$$
\text { als Controle der } \mathbf{X} \text {. Versuch gemacht. }
$$

Eine Maus wird nit $2^{1 / 2}-3$ etg Jodoform geimpft. Tol nach zwoi Tagen unter denselben Erscheinungen des Wahnsinns wie vorher. In der Tasche liegt noch freies Jodoform. Der T'od unterscheidet sich sehr vom Milzbrandtode.

\section{Controle. XI. Versuch.}

Eine Maus wird mit ca. 1 deg vergiftet. Tod nach 9 Stundell. In der Hauttasche Jodoform. Jch erwähne den Versuch deshalb, weil der Diener angab, dass die Maus sich auf Reize nur mit den Vorderpfoten fortzubewegen suchte, die Hinterextremitäten nachziehend. Es war also dureh Jodoformintoxication eille Lähmung der Hinterextremitäten eingetreten.

$$
\text { XII. Versuchsreihe. }
$$

$$
\text { Virulen\%. }
$$

Wachsthum.

$\left.\mathrm{XII}{ }^{2}\right) 9$ Tage alte Jodloform-Cultur wird geimpft. Maus In 3 Gläschen wachbleibt 6 Tage gesund. Am 7. Tage erfolgte der Tod sen schnell verflüssiil einer Lage über der Bauchfiäche gekrümmt. Seröses gende Coccen, aber Oedem der Bauchdecken und des Darms.

$$
\text { XIII. Versuchsreihe. }
$$

$\left.\mathrm{XIII}^{3}\right) 9 \mathrm{~T}$ Tage alte Cultur wird geimpft, 20 Tage beobachtet; Maus blieb ges und. XIV.

$\mathrm{XIV}^{2}$ ) Da Maus aus XII gestorben war, so wurde aus demselben Originalgläschen eine $15 \mathrm{Tage}$ alte Cultur ceimpft. Protoplasmatische Degeneration in hohem Grade. Maus 18 Tage beobachtet bleibt gesund.

$$
\text { XV. Versuch, modificirt. }
$$

Es wird ein $5 \mathrm{~g}$ Gelatine enthaltendes Röhrehen verflüssigt und mit 50\% Jorloform bestreut; gemischt und erstarren gelassen, so dass eine möglichst gleichmässige Vertheilurg des Jodoforms erfolgte. Sodann wurde sehr reichlich mit Milzbrand geimpft. Nach 6 Tagen war die schon nach drei Tagen bemerkbare punktförmige Verfüssigung weit fortgeschritten. Im Präparat sieht man Fäden mit protoplasmatischer Degeneration und daneben noch gut aussehende. Von dieser 7 Tage alten Cultur wird eine Maus geimpft. Dieselbe bleibt nach 10 tägiger Beobachtung g esund.

Zu gleicher Zeit wurden von dieser Anthrax-Gelatine 3 Platten angelegt. Es wuchsen wohl graue nicht verflüssigende Coceen, die uns oft die Culturen verunreinigten, aber kein Anthrax.

Ueberblicken wir diese Versuche, so kommen wir zu einem sehr bemerkenswerthen Resultat: Der Milzbrand mit Sublimat gemischt, hat seine für Mäuse tödtende Wirkung s of ort eingebüsst und erlangt sie nicht wieder; der Milzbrand hingegen, welcher mit Jodoform gemischt war, hat vom 1 . bis 5 . Tage seine Virulenz für Mäuse behalten, allein es ist deutlich eille Abschwächung zu constatiren. Diese Abschwächung der Virulenz ist nach dem 5. Tage b is zur Unschädlichkeit für Mänse gesunken. Zugleich sehen wir, dass trot $z$ der Verflüssigung im Originalgläschen die Fortpflanzungsfähigkeit auf neue Gelatine aufgehört hat; und zwar wachsen schon zn einer Zeit keine Milzbrandbacillen in neuer Gelatine mehr, wo Mäuse noch von denselben (nicht mehr auf Gelatine fortpflanzungsfähigen) Milzbrandbacillen getödtet werden. Diese sonderbare Thatsache ist nicht neu, auch $\mathrm{Koch}^{1}$ ) hat bei seinen Versuchen über die Ab-

1) Mittheilungen aus dem Kaiserlichen Gesundheitsant, II. Band, 1884. schwächung des Anthrax ganz dasselbe beobachtet. Er meint, dass noch lebensfähige Keime vorhanden gewesen sein müssen, und erklärt die Thatsache so, dass die Umsetzungsproducte der Bacillen diese selbst abtödteten. Wir glauben hier wohl annehmen zu mïssen, dass das Jodoform die Bacillen "abschwächt resp. abtödtet, und dass sie früher oder später in ein Stadinm der Abtödtung gekommen sind, dass siein demvorzüglichsten Boden, den wirkennen, d. i. in dem Organismus der Maus, noch gedeihen, in dem weniger guten Nährboden derGelatine sich nicht wieder zu neuem Wachsthum erheben können. -

Auch hier entsteht wie bei dem Wachtsthum wieder die Frage, wodurch wirkt das Jodoform auf die Bacillen? Ist es vielleicht blos eine Wachsthumsbehinderung, eine Abtödtung der Bacillen durch das trockene Jodoform, gerade so wie eine Abtödtung der Bacillen im trockenen Sandhoden erfolgt.? Mit anderen Worten: Sind die merkwürdigen Resultate nur dnrch die Trockenlegung durch Jodoform erfolgt, oder sind sie wirklich eine Wirkung des Jodoforms? Es wurden daher mit Sand Controlversuche gemacht. Die Bereitung der Sand-Anthrax- und Jodoform-Anthrax-Gelatine ist schon oben angegeben. Beide Male wurde eine 10 Tage alte Cultur, die die Gelatine fast ganz verflüssigt hatte, genommen, als rein und als virulent gepriift, sodann mit $40^{0} ; 0$ Sand resp. Jodoform beschickt. Der Theil der nicht verflüssigten Gelatine ist dentlich erkennbar, daher auch das Fortschreiten der Verflïssigung. Die Impfungen, d.h. immer subcutane Applicationen, geschahen unter der oben angegebenen doppelten Controle der Reinheit und der Einverleibung von Anthrax.

\section{Sand-Anthrax-Gelatine. \\ Virulenz. \\ Wachsth \\ Jodoform-Anthrax-Gelatine.}

I. Versuchsreihe (8 Experimente).

1 Tag alte Cultur Wachsthum von 1 Tag alte Cul- Abimpfung ab reimpft. Tod an Bacillen, aber tur geimpft sehr solut steril noch Milzbrand nach mit Coccent ver- reichlich. Nächstell nach 1 Monat.

$1^{1 / 2}$ Tagen. unreinigt.

\section{Tag keine Bacillen} im Sihwanzblut (sieh

Bemerkung unten.)

Tod nach neun Tagen.

Kein Milzbraudtod.

II. Versuchsreihe (8 Experimente).

2 Tage alte $\mathrm{Cul}$ - In allen 3 Gläs- 2 Tage alte $\mathrm{Cul}$ t ur geimpft. Tod chen Milzbrand- tur geimpft. Tod an Milzbrand nach $\begin{gathered}\text { wachsthum. } \\ \text { an Milzbrand nach }\end{gathered}$

2 T'agen. 4 Tagen.

III. Versuchsreihe.

3 Tage alte Cultur greimpft. 'Tod nach 20 Stunden.

Milzbrand
wachsthu

$$
\left|\begin{array}{c}
\text { 2) } 3 \text { T ag e al t e Cul - } \\
\text { tur geimpft, sehr } \\
\text { reichlich und ziem- } \\
\text { lich viel Jodoform. } \\
\text { Tod nach } 24 \text { St. nicht } \\
\text { an Milzbrand. }
\end{array}\right|
$$

IV. Versuchsieihe.

5 Tage alte Cultur geimpft. Tod nach 24 Stunden.

\section{\begin{tabular}{l|l} 
Milzbrand- & 5 Tage alte Cul-
\end{tabular}} Wachsthum $\quad$ tur geimpft. Maus V. Versuchsreihe.

10 Tage alte Cul-| Wachsthum.

tur geimpft. Die

Bacillen zeigen starke

Gliederung und Zer-

fallsproducte, da-

neben noch viele

gute Bacillen. Tod

an Milzbrand nach

$2^{1 / 2}$ Tagen.

Enorm viele Bacillen im Blut.

Wir miissen mit einigen Worten auf das 1. und 3. Experiment bei der Maus, die mit Jodoform-Anthrax geimpft ist, eingehen. Am 1. Tage nach der Impfung waren keine Bacillen im Schwanzblut zu constatiren, 8 Tage blieb die Maus am Leben. Beim Tode sieht sie aufgedunsen aus, Haare struppig in die Höhe ragend, Augen verschlossen und eitrig verklebt, Kopf und ganzer Körper enorm geschwollen. Von der in Agonie liegenden Maus wurden 2 Tropfen des lebenden Blutes ans dem Schwanze einer gesunden Maus eingeimpft. Mikroskopisch sind keine Bacillen zu finden. Die so geimpfte Maus blieb gesund noch nach 1 Monat, während die erstere Maus am Abend starb. Section: Die Schwellung des Körpers ist hervorgerufen durch ein enormes subcutanes, submuskuläres und intraabdominelles Oedem von trüber Flüssigkeit. Milz und Leber stark geschwollen. Im Blut, im Parenchym der Leber, Milz, Niere, kein einziger Bacill zu sehen. Es wird jetzt nach dem Tode von dem Blut und der Oedemflüssigkreit eine neue Maus geimpft. Dieselbe 
starb nach 7 Tagen unter den gleichen Erscheinungen. Es ist das, wie man sieht, eine andere Krankheit als Milzbrand. Darauf kommt es hier nicht at.

Was den 3. Versuch betrifft, so ist die Mans uach 24 Stunden gestorben; allein ich kann bei der grössten Sorgfalt diesen Tod nicht als Milzbrandtod bezeichnen. Die Maus lag 8 Stmolen wie todt da und wurde auch als todte rom Diener bei Seite gestellt. Als sic zur Section angenagelt werden sollte, bewegte sie sich, und es reigte sich offenbar, dass sie betäubt war. In der Hauttasclie noclı freies Jodoform. Kïnstliche Athemversuche stellten eine bessere Athrnutg her und nach $1 / 4$ Stunde ging die Maus wicder. Bei einem zu brïsk ausgeführten weiteren Athemversuch starb sie dann. Die Milz war nicht geschwollen, nicht geröthet, sonderut eher blass, kein serös-sulziges Oedem. Im Blut der Leber, Milz, des Hermons kein einziger Bacill zu constatiren. Drei Platten vom Herzblut sind steril geblicben. Der Tod ist wohl durch Jodoformintoxication crfolgt.

Die Versuche zeigen also, dass Sand-Anthrax noch nach zehn Tagen dieselbe heftige Virulenz besitzt, wie gewöhnlicher Milzbrand, dass also das Jodoform als Pulver, indem es etwa die Bacillen trocken legen könnte, nicht eine Einwirknng ausïbt. Wie dassclbe aber wirkt, bin ich aussce Stande zu sagen. Moglich ist es vielleicht, dass die Umsetzungsprodncte der Bacillen mit dem Jodoform Verbindungen eingehen, dasselbe zerlegend, und dadurch antibacterielle Kraft erlangen.

Es wurde roch die Frage gepriift, ob etwa verschiedene Me ngen des zugefügten Jodoforms einen verschicdenen Einfluss auf den Milzbrand ausübten. Die Versuclıe ergaben, dass es ganz gleichgïltig für dic Alschwächlung sei, ob mehr oder weniger Jodoform hinzugesetzt ist. Es sind diese Versuche nicht ausführliclı crwïhnt, um den Leser nicht zu verwirren, sondern ein Theil der Versuche ist unter die crste grosse Versuchsgruppe eingefïgt, und es ist dort (durch die Exponenten ${ }^{5}{ }^{2}{ }^{3}$ ) die Menge des Jodoforms angedeutet. Die Zall 1 bedeutet, dass zu der im Trichter verflüssigten Gelatine ca. ${ }^{1 / 4} \mathrm{~g}$ Jodoform linzugesetzt wurde, d. h. soviel, dass der Trichter ganz erfüllt und gemischt ist; bei 2 wurden zu ciner fast ganz verflüssigten $3^{1} / 2 \mathrm{~g}$ enthaltenden Anthrax-Gelatine $500 \mathrm{mg}$ zugesetzt; bei 3 wurde eine ebenso alte und gleiche Milzbrand-Gelatine-Cultur mit $1 \mathrm{~g}$ Jodoform beschickt. In alleı diesen Fällen wurde immer vor der Mischung die Virulenz an dem Tod einer Maus, das Wachsthum an der Ueberimpfung festgestellt.

Weil die Abschwächung der Anthrax-Bacillen durch Jodoform ebenso interessant als wichtig erschien, wurde folgende, schon oben beim Wachsthum erwähnte Versuchsreihe angestellt, welche dem Uebelstande der Verunreinigung anf einfache Weise answich. Es wurden nämlich, wie oben näher buschrieben, die in Bonillon geziilchteten Milzbrandbacillen mit je $1 \mathrm{~g}$ Jodoform beschickt, ebenso wurde eine Reihe Controlgläser angestellt. Die Impfung wurde unter denselben doppelten Cautelen, wie vorher, ausgeführt.

\section{Jodoform-Anthrax-Bonillon.}

\section{Virulen\%} Milzhrand nach 3 T agen.

B) 2 Tage alte Cultur geimpft. Tod der Maus an Milzbrand nach 3 'l'age 1 .

C) 3 Tage alte Cultur geimpft. Maus gestorben nach 4 Tagen. storben nach 2 $T$ a g $\in$ ll. torl uach 3 Ta $x$ en.

F) 6 Tage alte Cultur geimpft. Proto- F) Sterilität î allen Gläscru. plasmatische Degeneration ter Bacillen zu constatiren.

Tod an Anthrax nach 1 Tag. plasmatische Degentration. Tod an Anthrax, nach $1^{1}{ }_{2} \mathrm{Tag}$.

H) 8 Tagealte: Cultur geimpft. Mil\%brandtod nach 3 Tagen.

I) 9 'l'age alte Oultur geimpft. 'Tor nach 4 Tage

J) 10 Tage alte Cultur geimpft. Milzbrandtorl nach $2 \mathrm{~T}$ agen.
H) In keinent ter Gläschen 1 Wachsthum. Sterilität.
A) 1 Tag alte Cultur geimpft.

D) 4 Tage alte Culturgeimpft. Maus ge- D) In 2 Gläschen Sterilităt,

E) 5 Tage alte Cultur geimpft. Milshrancl-

G) 7 Tage alte Cultur geimpft. Proto- G) Kein Warhsthum in den
Diese Experimente, welche so wic dic vorhcrgegangenen mit allen möglichen Cantelen angestellt sind, geben ein gänzlich anderes Resultat, als die Versuche mit der Jodoform-Anthrax-Gelatiue. Wenn anch in 4 bis 5 Impfungern ein Hitansschieben des Todes un 1 bis 2 und 3 Tage nicht zh lengnen ist und damit also anch nicht eine gewisse Alsschwïchung der Viruleuz, so ist doch die cutibacterielle Wirkung des Jodoform auf don Milzbrand höchst gering. Wic lassen sich diese Differenzen erklärcen? Wir mö̈hten dafïr 3 Nomente beschuldigen. Zunächst wurden dic Bonillon-Gläsclen $3 \times 2+$ Stunden in deu Britapparat gestellt, und wenn anch der Thermostat auf $25^{\circ}$ gestellt war, so stelit es doch fest, dass mitunter, besonders in der Nacht, eine viel höhere Temperatur im Brutraum statt hatte, dercn Regulirmng meist dem Diener überlassen wurde. Musste doch am Tirge selur oft die Temperatur regnlirt werden Nun entwickeln sich leicht bei (iner Temperatur von $30^{\circ}$ Sporen. Als nach dew 5. Tage die Virulen\% noch nicht völlig vernichtet war, tuntersuchte ich jedes Gläsclien auf Sporen, bevor ich impfte, und in der That fund ich zweimal solcle; aher in den übrigen Malen komte ich trotz genaner Untersuchung keine nacliweisent Trotzdem aber ist die Möglichkeit der Sporenbildung rorhanden gewesen. Ein zwcites Moment liegt in der Boulllon selhst. Es gelassen. Die Bacillen steigen, weil sie viel leichter als das sich schnell жu Boden schlagende Jodoform siud, in div Höhe und lagern sich awischen Jodoform und der Bonillon. Nun entwickelu sie sicl weitcr, allerdings nur nach oben in die Bontllon hintein. Hätten wir jeden Tag umgeschïttelt und dic Cultur inniger mit dem Jodoform in Verbindung gebracht, so wäre vielleicht das Resultat anders ausgefallen. Bei der Golatine kommt das Jodoform viel lïnger und inniger in Berïhrung mit den Bacillen, das Jorloform scheidet sich viel langsamer aus, oft hleibt mehrere Tage noch iiher der klaren Gelatinte oben anf der Oberfläche ein Jodoformhäntchlıen. und dann wurde die Mischung öfter geschüttelt, weil sie öfter zur luppfung verwendet wurde. Es crhellt aus diesen Verhültuissert. dass dals Jodoform jedenfalls keinte gute Femwirkung ansïhe, sondern am besten local wirken müsse. Vielleicht ist es am richtiosten, diese Differenz in chemischon Verhältnissen zu suchen. Es ist möglich, dass das Jodoform durch die Bouillon garnicht chenisch alterirt wird, während das von der chemisch doch etwas differenten Gelatine geschieht.

Aber soviel geht auch aus dicsen Versuchen liervor, dals die Fortpflauzungsühigkeit anf nener Gelatine abgeschwächt, gehentut nud uach kur\%er Zeit völlig abgetöd tet wird. Würe dieser Satt uicht so offenbar ans den Imptungen mit dem Bonillon-Anthrax hervorgegangen, so würden wir überhaupt keine Jodoformwirknng anf die Milzhrundhacillen annelmen, wenn diese in Bouillon gezüchtet sind. Auch hier zeigte sich wic bei der Gelatine, dass, während der Milzbrand in der nenen Gelatine. aus der Bouillon genommen, nicht mehr wächst, doclı die Mäus, getödtet wurden. Anch hier gilt obige Erklärung. -

Alle bisherigen Versuche sehen wir als solche ausserhalb des Tlierkörpers angestell te an. Freilich ist mit den Bacillen immer etwas Jodoform mit in die Hauttasche gekommen, und gewiss entfaltet dieses hier fïr sich seine antibacterielle Wirknng; haben wir doch sogar den Tod zweier Mäuse als Jodoformtod erklïren müssen. Allein die allmähliche Abschwïchung der Virulenz, die protoplasmatische Degeneration, die Ueberpflanzungseresultate zeigen doch nuzweife:haft eine Wirkung ansserhalb des Organismes an. I)emoch aber wurde roch in eincr Reihe von Versuchen geprïft:

\section{Wie wirkt das Jodoform innerhalb des thierischen Organismus auf den Milzbrand?}

Wirkt es local oder allgemeiu?

Wirkt das Jodoform local?

Einc bestimmte Menge Jodoform wird in cinc Hanttasche gebracht und nach bestimmten Zeitintervallen Milzbrand. der vorher int tiner Maus als virulent geprüft war. Es sollte znerst mit getlanen Centigrammen Jodoform experimentirt werden. Da die Abwägung so kleiner Gewichte bei dem schweren Jodoform schwierig war, so liessen wir uns mehrere Pulver à 1 degr abwägen. Jedes Pulver worde gleichmässig vertheilt und nach dem Angenmaass 1, 2, 3 Zehntel genommon. Wiewohl ja. wie wir oben gefurtcu, die Yenge des Jodoform keinen Einfluss anf die Bacillen hat. so ist dieselbe für die kleinen Mäuse nicht gleichgültig. Aus mohrercn diesbezüglichen Versuchen haben wir gefunden, dass eine weisse Maus im Durchischnitt 2 ctgr. erträgt; allerdings sterben unanche schon an viel kleineren Dosen. Dieses muss man bei dem Tode der Yäuse immer berücksichtigen: Es wirkeu auf die Gesundlutit zwei arge Feinde ein: ein chentisches Gift und ein hoftiges organisirtes, und gewiss werden dadurch viele Differenzen zu erklären sein. - Es wurden also immer c. 2 ctgr. Jodoform in die Hauttasche gcbracht. Sodant wurden die Versuche nngekehrt gentacht: in eine und dieselbe Hautwurden dic Gläschen nur eimmal umgeschüttclt und datn stchen 
tasche wurde zuerst Authrax geimpft und dann nach bestimmten Zeitintervallen Jodoform. Milzbrand wurde immor in enorm grossen Quantitäten geinipft.

In eine und dieselbe Hauttasche einer weissen Maus

werden 2 ctgr. Jorloform gubracht $\mid$ werden sehr grosse Anthraxmengen geund nachher sehr reichlich Anthrax bracht und nachher 2 etgr. Jodo.

\begin{tabular}{c|c} 
und zwar: & form u. zwar \\
Torl nach & Tod nach
\end{tabular}

nach Stl. Tagen Bemerkungen. nach Sttl. Tod Tagon Benerkungen.

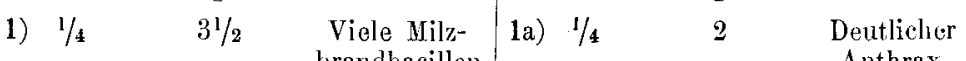

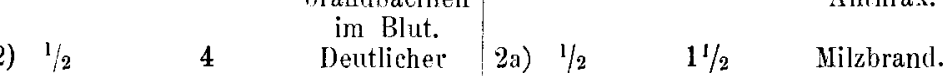

3) 1 bleibt

12t tăgige Beob- 3a) $1 \quad 4^{1 / 2} \quad$ Sehr wenig Bagesund achtung.
sillen im Blut,
starkes Oedem.

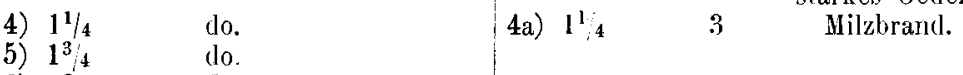

6) ${ }^{13}$

(Controle zu 6)

7) 2 bleibt gesund

siche untel.

8) 3 Ho

7a) 2 2 Milzbrand.

9) Einem grossen und starken grauen Kaninchen wirl 3 (lcgr. Jodloform in eine Hanttasche gebracht, nach 24 Stunden nochmals in dieselbe Tasche 1 degr. und nach 3 Stil. darauf eine ungeheller grosse Menge einer virulenten Anthraxcultur. Das Kaninchen blieb gesund.

Versuch 6. 7, 8 verdient noch einige Worte. Die Maus 6 starb llach 3 Tagen. In der Hanttasche ist aber keine Spur voll Jodoform mehr enthalten; im Blut wenige Milybrandbacillen. Inı Leber-, Milz- und Herzblut kein Jod mit Stärkepapier nachzuweisen. Es ist wohl anzunehmen, dass zul wenig Jodoform genounmen wirde; als dieses anfgesogen war, konnten dic: Bacillen sich weiter vernuehreıl. Bei 7 ind 8 wurdell daher grössere Melgen, dem Angenmaass zufolge 3 bis 5 ctgr. gelıommen. Ferner wurde zu diesen Versuchen eine Milzbrandcultur von Agar benutzt, welche laut mikroskopisclier Untersuchung dentliche Sporen enthielt.

Wir sehell also, dass das Jodoform im thierischell $\mathrm{Or}$ ganisnus eine ganz bedeutende antibacterielle Kraft besitzt. Delln es giebt wohl kein heftigeres Gift für die Mäuse als den Milybrand; allerdings ist die Wirkung des Jodoforms keine augenblickliche, sondern eine allmähliche, und daher ist.Jodoform erst nach $1 / 2$ stïncligem Aufenthalt in thierischen Organismus im Stande, die eillgeimpften Milzbrandbacillen unschädlicll zu machen. Hat dagegcn der Milzbrand nur $1 / 4$ Stunde Zeit, ungestärt ill der Malls sich zu vermehron, so kann kein Jodoform mehr den Milzhrandtod verhïten; selhstverständlich ist eine Verhütıng des Todes $1 \mathrm{~m}$ so schweler, je läloger der Milzbrand in Körper olne Jodofor'm gelassen wurdc. abel es lässt sich anch hier nicht verkelmen, dass der Tod kraft der antibacteriellen Jodoformwirkung cin wenig hinausgeschoben wird. Ich habe dieses Hinausschieben des Todes deshalh imoner so scharf urgirt, weil dieses hei wcniger für Milzbrand empfänglichen Thieren giinstiger wirken könnte. Die Versuchc lehren ferner das nicht nnwichtige Factum kennen: Wenn die Milzbraudbacillen einmal in's Blut ïbergegangen sind. wozu. wie das obige Expeliment zeigt, höchstens 1/4 Stunde nöthig ist, so ist keine locale Jodoformmenge mehr im Stande, die deletäre Verinehrung der Bacillen zu verhïten. Wenn wir aber dieses wissen, und ferner, dass das Jodoform erst nach 1/2 Stunde dic Mil\%blandbacillen im Körper zu tödten im Stande ist, vorher aber diese sich trotz der Anvesenlueit des Jodoforms vermchren und in's Blut übergehen, so erwächst jedem praktischen Chirlloren die Pflicht, falls el das Jodofol'm auf eine frische Wunde streuell will, dieselbe volher dulch eill sofolt wirkendes Alltibactericum wie Sublimat von Pilzell, oder besser ausgedräckt voll pathogenen Pil\%cr, frei zu machen. Nan dïrfte umsomehr nicht eine septische Wundfläche nur mit Jodoform bestreuell. weil schr gut nnter sonst gülstigen Verhältnissen die cinige Zeit voll dcm Jodoform nicht alterirten Bacterien in's Blıt übergehen könuen, und dann das Jodoform kaum mehr wirken wirl. Freilich sind die Bactcrien der Fäulniss und der Eiterung lange nicht so gefälırlich wirkend, wie der Milzbrand, und darum sind die Verhältnisse doch ein wenig andere, $d, h$. für .Jodoform günstiger liegende. Man wird aber jedenfalls doch verpflichtet sein, jede bacterielle Wunde vorher nit Sublimat zu behandeln. - Es dürfte anch in der 'That wenige Chilurgen geben, welche nicht schon diesen durch neine Untersichungen gebotenen Forderungen seit langer Zeit gerecht geworden sind.

Es blieb nur noch ïbrig, zu prïfen, ob das Jodoform auch allgemein wirkt.

Obwohl durch die vorherigen Velsuche dies mit einigem Recht verueint werdeu konnte, wurden Mäusen je 2 entfernte Hanttasclien, die nicht mit einander communicirtell, angelegt. In die eine kommt 3 ctgr. Jodoforin, in lie andere reichlich Milzbrand. Dic Mäuse starben alle an Milzbrand. Allein das kann uns nicht Wunder nehmen, da ja einerseits schon 1/4 Stunde nach der Milzbrandimpfung Bacilleı in's Blut übergegangeu sind, andererseits das Jodoform erst nach $1 / 2$ Stunde die Bacillen zu tödten beginnt, inithin die Bacillen mindestens $1 / 4$ Stunde Zeit hatten, sich $z u$ verinehren und in's Blut iberzugehen. Es wurden daher diese Versuche variirt dergestalt, dass das Jodoform 1-3 Std, vorher eingeimpft wurde, so dass es sicher eine Zersetzung und eine antibacterielle Wirkung entfalten könne, und dann erst wurde in eine zweite Hauttasche reichlich Antlıax geimpft. Die Mäuse starben alle in 1 bis 3 Tagen an Milzbrand. Es wurde schliesslich Mäusen eine starke .Jodoformintoxication beigebracht und dann nit Anthrax geimpft, anch dann starben sie. Wir könllen also sicher behallpten, dass die antibacterielle Wirkung des Jodoforms nur eine locale und keine allgemeine ist. -

Für den, welcher die Immnnitätsversuche vou $\mathrm{Koch}$ kenut, lag der Gedanke, ob etwa die von Jodoform-Anthrax verschont gebliebellen Mäuse fül eine zweite Impfulı mit virulentem Milzbrand immun seien, sełır nahe. Man brauchte blos sämmtliche gesund gehliebenen Mäuse mit Milzbrand zu impfen. lch habe das bei einigen gethan und gebe die folgende Tabelle: Auch hier wurde mit allen Cautelen die subcutane Application angewandt. Bei Maus 2 wurden Sporen geimpft, bei den anderen Bacillen, bei 4 frisches Milzhrandblut.

$$
\text { Tabelle. }
$$

1) 7 'Tage gestund gebliebene Maus stirbt nach 4 Tageu an Anthrax.

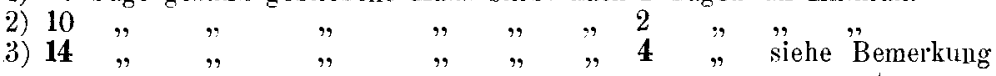

$$
\begin{aligned}
& \text { 4) } 15 \quad, \quad, \quad, \quad, \quad, \quad, \quad, \quad, \quad, \quad \text { anl Anthrax. }
\end{aligned}
$$

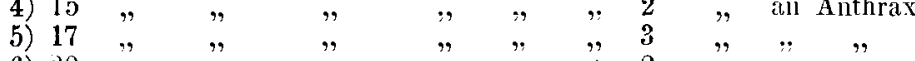

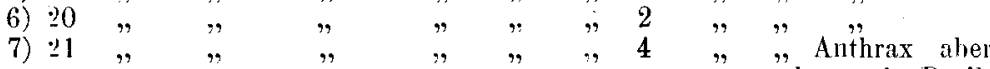

$$
\begin{aligned}
& \text { sehr wenig Bacil- } \\
& \text { len im Blut, Organe } \\
& \text { fast nicht ge- } \\
& \text { schwollen. }
\end{aligned}
$$

In dem 3. Versuche starb die Maus nach 4 Tagen. Es fanden sich aher in Herwblut, in der Juber, Mils, Niere, obwohl viele Präparate hergestellt wurden, keine Bacillen. Aber auch wenn die Maus an Milzbrand gestorben wäre, was ich nicht aunelunen kalın, so ist doch das Eintreten des Todes nach 4 'Tagen beinerkenswerth. Auch der 1. und 7. Versuch, in denen die Nüuse an Milzbrand gestorben sind, ist ebenso interessant. Ich muss hier wieder darauf zurück kommen, dass wenigel enıfäıgliche Thiere, als Mäuse, vielleicht ein günstigeres Resultat liefern werden. Belłanntlich gelang es auch $\mathrm{Koch}$ hei seiıen Immunitätsversuchen nicht, die Mäuse durch vorlier eingeimpften Milyhand, welcher dnrch eille Teinperatur von 42,6 $\mathrm{C}$ alogeschwächt war, gegen eine frische lufection mit virulentem Milzbrand immuı zu machen, dagegen gelang dieses bei einer Anzahl von Hammeln etc. Ich hielt es für gebotel, einfach diese Resultate der Immunitätsversuche mit meineı Mäusen anzılï̈hren, weil vielleicht ein Anderer, der melı Zeit und Gelegenheit loat, als ich jetzt, die Versuche bei antleren Thieren wciter fortfïhrt. Jedenfalls muss man zugeheu, dass bei manclien Näısen cin Effect des vorher eilgeimpften Anthrax-.Jodoform für eine spätere Infoction unverkennbar ist.

Es dürfte sich empfehlen, a.n Schluss diesel Arbeit die erlangten Resultate der Haltptsache nach zusanmen- zustellen. Dieselben wiilden etwa folgende sein:

1. Es giebt eine Vesflüssigung der Gelatine durch Bactericn ohne Wachsthum der letzteren. Die Verflüssigung erfolgt wahrscheinlich durch chemischt: Verbindungen, deren eine Componente ein Unisatzprodict der Bacillen sein muss.

2. Dic Einwirkung des Jodoforms auf die Milshrandbacillen zeigt sich:

a) physiologiseh in del Heinmung des Wachsthums und dem Aufhören der Fortpflanzungsfähigkcit und endlich in der Abschwäclıung der Virulenz, welche bis zur Unschädlichkeit fïr Mäuse geht.

b) irrorplo ologisch in $\mathrm{del}^{*}$ sog. ,protoplasmatischen Degeiıelation" der Bacillell.

3. Die Einwirkung des Jodoforms auf die Bacillen ist keille mechanische, solldeln einc chemische.

4. Innerhalb des thierischen Organismus entfaltet das Jodoform eine energische antibacterielle Wirkung. Dieselbe erfolgt indess nicht sofort nach der Application, sondern erst allmählich nnd $\mathrm{nach}$ einer gewissen Zeit.

5. Dic Einwirkung des Jodoforms auf die Bacillen innerhalb des Kölpers ist nur eine locale, nicht eine allgemeine. 
Am Schluss dieser Arbeit habe ich noch die Pfiicht, Herm Dr. Weigert für seine mir fortgesetzt bewiesene Liebenswürdigkeit aufrichtig auch hier zu danken. Er hat mir nicht nur sein Privat-

laboratorium, sondern auch sämmtliches todte und lebendige Mate-

rial zur Verfügung gestellt und oft bei den Untersuchungen mit Rath

und That geholfen. Ohne seine Mitwirkung und Hilfe hätte ich niemals die zahlreichen Versuche anstellen können. 